\title{
Temperature Dependence of Bulk Viscosity in Edible Oils using Acoustic Spectroscopy
}

Sunandita Ghosh*, Melvin Holmes and Malcolm Povey

Department of Food Science and Nutrition, University of Leeds, Leeds, UK

\begin{abstract}
When ultrasound waves are applied to a compressible Newtonian fluid, bulk viscosity plays an important parameter to cause attenuation. Ultrasound spectroscopy is an important technique to characterise and determine the physico-chemical properties of many food components because it is a non-invasive, non-destructive, easy and accurate technique. The aim of this study was to find the bulk viscosity of three brands of sunflower and extra-virgin olive oil by using the Navier's-Stoke equation across a temperature range of $5^{\circ} \mathrm{C}$ to $40^{\circ} \mathrm{C}$ and to test the hypothesis that there is a significant difference in the value of bulk viscosity between the different brands of sunflower and olive oil used. The value of bulk viscosity was not found to be constant over the operating frequency range of $12-100 \mathrm{MHz}$, which suggested edible oils are non-Newtonian fluids. Also, no significant statistical difference of bulk viscosity values was found between different brands of the same oil ( $p \geq 0.05)$. This shows bulk viscosity is not affected by small compositional variations. Acoustic spectroscopy is increasingly being used to characterise food materials. More studies on bulk viscosity must be employed in order to be able to utilise this technology to its full strength.
\end{abstract}

Keywords: Bulk viscosity; Acoustic spectroscopy; Attenuation

\section{Introduction}

Edible oils occupy an important position in the human diet because of its nutritive value and also because of its organoleptic and rheological properties [1]. Acoustic spectroscopy is increasingly being used to characterise oils and fats due to its many advantages. Ultrasound waves are longitudinal sound waves of frequency of $20 \mathrm{KHz}$ or more [2]. Ultrasonic analysis is a useful technique to characterize and determine many physico-chemical properties of oil and other food component mainly because it is a non-invasive, non-destructive, easy and accurate technique. It can be used 'on-line or off-line' and also works for opaque food objects where characterising food on visual methods can be difficult [1].

Compressible fluids are fluids whose density changes when highpressure gradient is applied. When force is applied to these fluids, they flow in the form of transverse-pressure waves. The velocity of propagation of these pressure waves in compressible fluid is known as velocity of sound [3]. Newtonian fluids are ones whose shear stress is proportional to shear strain. Compressible Newtonian liquids exhibit two types of viscosity: shear and bulk viscosity. Shear viscosity is the resistance to the change in shape under shear stress and the bulk or volume viscosity is the resistance to change in volume under an applied pressure [4]. Bulk viscosity is also termed as volume viscosity, second viscosity coefficient, expansion coefficient of viscosity and coefficient of bulk viscosity. Bulk viscosity is significant if the compression or expansion in the fluids proceeds so rapidly that it takes longer time than the duration of change in volume to restore the thermodynamic equilibrium like with the absorption or dispersion of sound waves [5]. The experimental values obtained for ultrasonic absorption is often found to be much larger than the values obtained from classical Stroke's equation where only shear viscosity is considered [4]. This increase in the absorption can be attributed to the bulk viscosity and thermal conduction. Fluid molecules have translation, rotational and vibrational degrees of freedom. The translational motion is due to the dynamic viscosity and the rotational and vibrational motion is due to bulk viscosity. Therefore, to know the effect of vibrational and rotational energy on fluids obtaining the bulk viscosity data is very important $[5,6]$. There is also a certain bulk viscosity effect in fluid flow for fluids with large Reynold's number when the ratio of bulk to shear viscosity is of the order of the square root of Reynold's number [7]. However, given the importance of bulk viscosity little research has been conducted to characterise it and for many fluids it is unknown or inaccurately known particularly across different temperatures. Bulk viscosity is observed when sound particularly ultrasonic waves travels through fluids. Hence, ultrasound waves can also be used to measure the bulk viscosity of fluid.

In a study by Dukhin and Goetz [6], three methods were used to find the bulk viscosity: Brillouin spectroscopy, laser gradient spectroscopy and acoustic spectroscopy. It was found that the acoustic spectroscopy gave the most precise results for bulk viscosity; as with Brillouin spectroscopy there were 'high errors due to the difficulty in measuring the Brillouin linewidth' and with laser gradient spectroscopy complications arrived due to the fitting of the laser gradient with five adjustable parameters. Acoustic spectroscopy gives value for the speed of sound which can be used to measure compressibility and it is also the only method where multi-frequency measurements, in the range of $1-100 \mathrm{MHz}$ can be taken. This is important to find the nature of the fluid, if it's a Newtonian or non-Newtonian fluid. If the fluid is Newtonian than the calculated bulk viscosity will be independent of the frequency changes [6].

The Navier-Stokes equation is important to study physical fluid dynamics. The general Navier-Stokes equation is written as:

$$
\frac{\partial v}{\partial t}+(\mathbf{v} \nabla) \mathbf{v}=-\frac{1}{\rho} \nabla P+\eta \nabla^{2} \mathbf{v}+\frac{1}{\rho} \mathrm{F}
$$

Where $\rho\left(\mathrm{kg} \cdot \mathrm{m}^{-3}\right)$ is the density, $\mathrm{t}(\mathrm{s})$ is time, $\mathbf{v}$ is the velocity vector,

${ }^{*}$ Corresponding author: Sunandita Ghosh, Department of Food Science and Nutrition, University of Leeds, Leeds, UK, Tel: +44-0113-3437594; E-mail: sunandita.ghosh11@gmail.com

Received May 09, 2017; Accepted May 26, 2017; Published June 02, 2017

Citation: Ghosh S, Holmes M, Povey M (2017) Temperature Dependence of Bulk Viscosity in Edible Oils using Acoustic Spectroscopy. J Food Process Technol 8 : 676. doi: 10.4172/2157-7110.1000676

Copyright: $\odot 2017$ Ghosh S, et al. This is an open-access article distributed unde the terms of the Creative Commons Attribution License, which permits unrestricted use, distribution, and reproduction in any medium, provided the original author and source are credited. 
$\mathrm{P}(\mathrm{Pa})$ is pressure, $\eta(\mathrm{Pa} . \mathrm{s})$ is shear viscosity and $F(\mathrm{~N})$ is the body force term as such forces act on the volume of a fluid particle.

Bulk viscosity is an important term in the Navier-Stokes equation for a Newtonian compressible liquid [6].

$$
\rho\left[\frac{\partial v}{\partial t}+(\mathbf{v} \nabla) \mathbf{v}\right]=-\operatorname{grad} P+\eta \Delta \mathbf{v}+\left(\mu+\frac{4}{3} \eta\right) \operatorname{grad} \operatorname{div} \mathbf{v}
$$

$\mu$ (Pa.s) is the bulk viscosity. For an incompressible liquid, the last term on the right hand side may be neglected as this term accounts for compressibility.

\section{grad div $\mathbf{v}=0$}

Thus, the bulk viscosity term has no contribution for incompressible fluids. Therefore, for incompressible fluids the Navier-Stokes equation can be written as:

$$
\rho\left[\frac{\partial v}{\partial t}+(\mathbf{v} \nabla) \mathbf{v}\right]=-\operatorname{grad} P+\eta \Delta \mathbf{v}
$$

Thus the effect of bulk viscosity is not very significant for incompressible fluids and for ideal monoatomic gas for which $\mu=0$ [5].

When a wave propagates through a viscous and thermally nonconductive fluid then the general solution obtained from the NavierStokes equation with respect to attenuation is [6]:

$$
2\left(\frac{\alpha_{\text {long }} v}{\omega}\right)^{2}=\frac{1}{\sqrt{1+t^{2} \omega^{2}}}-\frac{1}{\sqrt{1-t^{2} \omega^{2}}}
$$

Where $\alpha_{\text {long }}\left(\mathrm{Np} . \mathrm{m}^{-1}\right)$ is ultrasound attenuation coefficient, $\mathrm{v}$ (ms $\left.{ }^{1}\right)$ is the velocity of sound, $\omega$ is the ultrasound frequency, $t(s)$ is the viscous relaxation time and takes into account both bulk and shear viscosity and is given by:

$$
t=\frac{1}{\rho v^{2}}\left(\frac{4}{3} \eta+\mu\right)
$$

If attenuation is plotted as a function of frequency a normal distribution curve is obtained and at the critical frequency the maximum value obtained is approximately equal to the viscous relaxation time. The critical frequency is around $1000 \mathrm{GHz}$ around, this high ultrasound range is difficult to achieve in real instruments but for low frequency this is achievable. The low frequency asymptotic function is given by [8]:

$$
\alpha_{\text {long }}=\frac{\eta \omega^{2}}{2 \rho v^{3}}\left[\frac{4}{3}+\frac{\mu}{\eta}+\frac{(\gamma-1) \tau}{\eta C_{p}}\right]
$$

Where $\gamma$ is the ratio of specific heats, $\tau\left(\mathrm{w} \cdot \mathrm{m}^{-1} \cdot \mathrm{K}^{-1}\right)$ is the thermal conductivity and $C_{0}\left(\mathrm{~J} . \mathrm{K}^{-1}\right)$ is the specific heat at constant pressure, $v(\mathrm{~m} . \mathrm{s}$ $\left.{ }^{1}\right)$ is velocity, $\mathrm{T}\left({ }^{\circ} \mathrm{C}\right)$ is temperature, $\beta\left(\mathrm{K}^{-1}\right)$ is the bulk compressibility and $\omega$ is the angular frequency $\omega=2 \pi \mathrm{f}, \mathrm{f}(\mathrm{Hz})$ is the frequency of acoustic wave and $i$ is the imaginary number. From equation (6) can be expressed as equation (7) to calculate bulk viscosity.

$$
\mu=\frac{2 \alpha \rho v^{3}}{\omega^{2}}-\frac{4 \eta}{3}-\frac{(\gamma-1) \tau}{C_{p}}
$$

$\alpha(\mathrm{Np} . \mathrm{m})$ is the attenuation coefficient. The contrihition of thermal conduction to bulk viscosity is dependent on $(\gamma-1) \cdot$ For liquids the ratio of specific heats is close to one and for gases it is greater than one as liquids are less compressible as compared to gases. Therefore, there is not much contribution to the bulk viscosity from the thermal properties of the material and the thermal term can be neglected [6].
Hence equation (7) can be re-written as:

$$
\mu=\frac{2 \alpha \rho v^{3}}{\omega^{2}}-\frac{4 \eta}{3}
$$

The temperature dependence of physical parameters of edible oils is demonstrated by the following model equations [9]:

$$
\begin{aligned}
& c=\mathrm{c}_{0}+\mathrm{c}_{1} \mathrm{~T} \\
& \rho=\rho_{\mathrm{o}}+\rho_{1} \mathrm{~T} \\
& \eta=\eta_{o} \exp \left[-\frac{\eta_{1}}{k(T+273.13)}\right]
\end{aligned}
$$

Where $c\left(\mathrm{~m} . \mathrm{s}^{-1}\right)$ is velocity, $\rho\left(\mathrm{kg} \cdot \mathrm{m}^{-3}\right)$ is density, $\eta\left(\mathrm{kj} \cdot \mathrm{mol}^{-1}\right)$ is viscosity, $\mathrm{k}$ is Boltzmann, $\mathrm{T}$ is temperature $\left({ }^{\circ} \mathrm{C}\right)$. The subscripted terms are constants. For sunflower oil, the values of the constants have been reported as: $\rho_{0}\left(\mathrm{~kg} \cdot \mathrm{m}^{-3}\right)$ is $933.76, \rho_{1}\left(\mathrm{~kg} \cdot \mathrm{m}^{-3},{ }^{\circ} \mathrm{C}^{-1}\right)$ is -0.61 for 20 to $80^{\circ} \mathrm{C}$; ln $\eta_{\text {is }}-13.83, \eta_{1}\left(\mathrm{~kJ} \mathrm{~mol}^{-1}\right)$ is 27.17 for $25^{\circ} \mathrm{C}$ to $50^{\circ} \mathrm{C}$; $\mathrm{c}_{0}$ (m.s $\left.{ }^{1}\right)$ is 1538 and $\mathrm{c}_{1}\left(\mathrm{~m} \cdot \mathrm{s}^{-1},{ }^{\circ} \mathrm{C}^{-1}\right)$ is -3.28 for $5^{\circ} \mathrm{C}$ to $70^{\circ} \mathrm{C}$. For olive oil $\mathrm{c}_{0}$ $\left(\mathrm{m} . \mathrm{s}^{-1}\right)$ is 1528.9 and $\mathrm{c}_{1}\left(\mathrm{~m} . \mathrm{s}^{-1} \cdot{ }^{\circ} \mathrm{C}^{-1}\right)$ is -3.23 for $20^{\circ} \mathrm{C}$ to $70^{\circ} \mathrm{C}$. All the three parameters velocity, density and shear viscosity are temperature dependent (equations 9-11) and plays an important role in finding bulk viscosity. Also, Coupland and McClements [9] emphasised that these bulk properties of oils depend upon their chemical composition. Therefore, there is expected to be a dependence of bulk viscosity on temperature for edible oils which will be discussed in this work (Figure 1).

The aim of this work was to calculate the bulk viscosity of sunflower oil and extra-virgin olive oil across a temperature range of $5^{\circ} \mathrm{C}$ to $40^{\circ} \mathrm{C}$ and to test the hypothesis that there is a significant difference in the value of bulk viscosity between the different brands of sunflower (Tesco, Morrisons, Floras) and extra-virgin olive (Tesco, Morrisons, Sierra mágina) oil. Even for the same type of oil there are differences in the physico-chemical properties as the composition of food oil varies significantly depending on the geographical source, processing parameters (like distillation), storage time (as crystallisation or oxidation might take place) [9]. The length of fatty acid chain has an effect on the viscosity of the oil [10]. Hence, three different brands were investigated to find if these differences have any significant effect on bulk viscosity. The experimental procedure of conducting the study is mentioned in the next section. The results obtained from this work is illustrated in the Results and Discussion part. First, the justification of using the frequency squared equation (8) for finding the bulk viscosity was given using the graph of $\log$ attenuation $\mathrm{v} / \mathrm{s} \log$ frequency (Figure 2). Second, all the bulk properties like velocity, density, shear viscosity and bulk viscosity was tabulated in Table 1 and the dependence of bulk viscosity on the frequency was studied (Figures 3 and 4 ). Third,

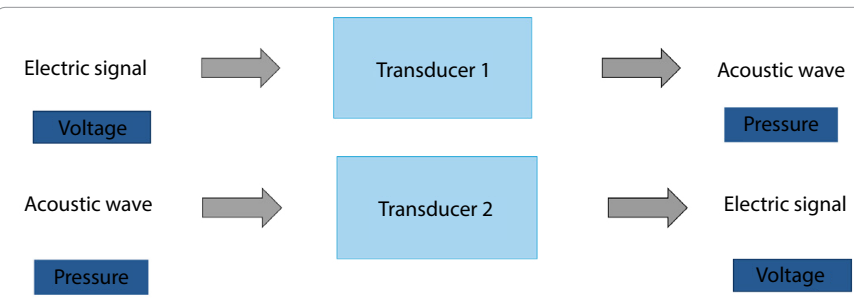

Figure 1: The first type of transducer emits ultrasound wave on application of electrical voltage which is picked up by the sample. The second type of transducer detects the ultrasound wave emitted by the transducer and converts it into electrical voltage which is measured as attenuation. 
Citation: Ghosh S, Holmes M, Povey M (2017) Temperature Dependence of Bulk Viscosity in Edible Oils using Acoustic Spectroscopy. J Food Process Technol 8: 676. doi: 10.4172/2157-7110.1000676

Page 3 of 6

a comparative study between the bulk and shear viscosity was made (Table 2 and Figure 5). Fourth, the hypothesis was tested to find out if

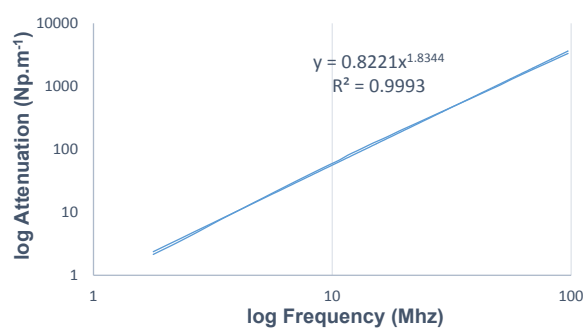

Figure 2: Plot of log Attenuation v/s log Frequency of Tesco sunflower oil at $25^{\circ} \mathrm{C}$ showing a best fit polynomial.

\begin{tabular}{|c|c|c|c|c|c|c|}
\hline Temperature & Density & Velocity & Shear viscosity & \multicolumn{3}{|c|}{ Bulk viscosity } \\
\hline \multirow{2}{*}{$\mathrm{T}\left({ }^{\circ} \mathrm{C}\right)$} & \multirow{2}{*}{$\begin{array}{c}\rho \\
\left(\mathbf{k g ~ m}^{-3}\right)\end{array}$} & \multirow{2}{*}{$\begin{array}{c}v \\
\left(m^{-1}\right)\end{array}$} & \multirow{2}{*}{$\begin{array}{c}\eta \times 10^{-2} \\
\text { Pa.s }\end{array}$} & \multicolumn{2}{|c|}{$\begin{array}{c}\mu \times 10^{-2} \\
\text { Pa.s }\end{array}$} & \multirow[t]{2}{*}{ CV } \\
\hline & & & & Mean* & SD & \\
\hline 6 & 928.71 & 1515.69 & 5.22 & 5.79 & 1.89 & 0.33 \\
\hline 10 & 926.01 & 1501.93 & 4.75 & 4.51 & 1.59 & 0.35 \\
\hline 15 & 922.57 & 1485.51 & 4.15 & 3.57 & 1.43 & 0.22 \\
\hline 20 & 919.15 & 1469.02 & 3.55 & 2.91 & 1.21 & 0.41 \\
\hline 25 & 915.74 & 1452.04 & 2.37 & 3.33 & 0.99 & 0.3 \\
\hline 30 & 912.35 & 1435.36 & 2.1 & 2.77 & 0.86 & 0.31 \\
\hline 35 & 908.97 & 1419.2 & 2.06 & 2.07 & 0.71 & 0.2 \\
\hline 40 & 905.6 & 1403.98 & 1.42 & 2.36 & 0.6 & 0.25 \\
\hline
\end{tabular}

Standard Deviation (SD)

Coefficient of Variation (CV)

"Mean was taken for the bulk viscosity in the frequency range of $12 \mathrm{MHz}-100 \mathrm{MHz}$ Ultrasound velocity readings was obtained from the Ultrasizer. Density was measured by Anton Paar DMA $4500 \mathrm{M}$ density-meter and shear viscosity by Anton Paar MCR 302 rheometer.

Table 1: Density, velocity, shear viscosity and mean bulk viscosity of tesco sunflower oil at the selected temperatures.

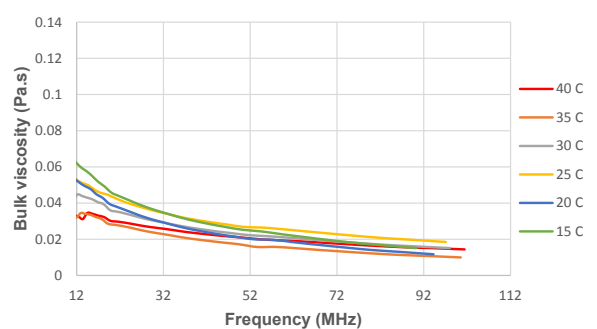

Figure 3: Plot of bulk viscosity v/s frequency of Tesco sunflower oil at each selected temperature. A decrease in the value of bulk viscosity is seen with the increase in temperature, showing frequency dependence and possible nonNewtonian behaviour.

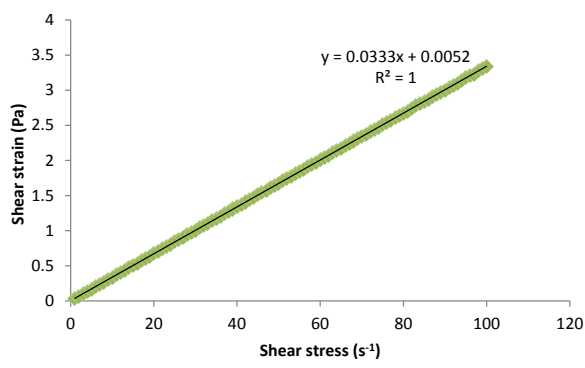

Figure 4: Plot of shear stress v/s shear strain, giving a linear relationship between stress and strain suggesting Newtonian behaviour of edible oils.

\begin{tabular}{|c|c|c|c|c|}
\hline Temperature & \multirow{2}{*}{\multicolumn{2}{|c|}{$\begin{array}{c}\text { Bulk viscosity } \\
\mu \times 10^{-2} \text { Pa.s }\end{array}$}} & \multirow{3}{*}{$\begin{array}{l}\text { Shear viscosity } \\
n \times 10^{-2} \text { Pa.s }\end{array}$} & \multirow{3}{*}{ Ratio $\left(\frac{\mu}{\eta}\right)$} \\
\hline \multirow{2}{*}{ 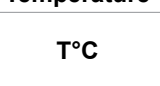 } & & & & \\
\hline & Mean & SD & & \\
\hline 6 & 5.79 & 1.89 & 1.89 & 1.11 \\
\hline 10 & 4.51 & 1.59 & 1.59 & 0.95 \\
\hline 15 & 3.57 & 1.43 & 1.43 & 0.86 \\
\hline 20 & 2.91 & 1.21 & 1.21 & 0.82 \\
\hline 25 & 3.33 & 0.99 & 0.99 & 1.41 \\
\hline 30 & 2.77 & 0.86 & 0.86 & 1.32 \\
\hline 35 & 2.07 & 0.71 & 0.71 & 1 \\
\hline 40 & 2.36 & 0.6 & 0.6 & 1.66 \\
\hline
\end{tabular}

Table 2: The bulk and shear viscosity values of Tesco sunflower oil at the selected temperatures and the ratio between bulk viscosity to shear viscosity.

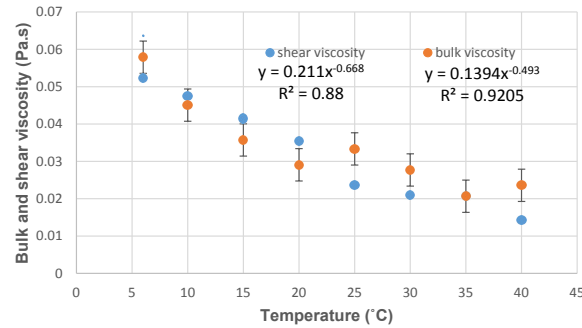

Figure 5: Plot of bulk and shear viscosity v/s temperature of Tesco sunflower oil showing a decrease in the values with the increase in the temperature.

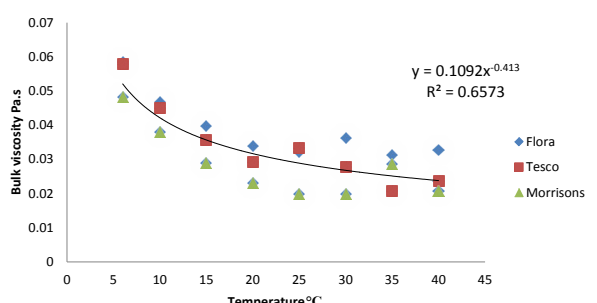

Figure 6: A plot of bulk viscosity v/s temperature of all the three brands (Tesco, Morrisons, Flora) of sunflower oil. As there is no significant difference between the brands an averaged polynomial best fit is obtained from this plot to know the temperature dependence of bulk viscosity for sunflower oil.

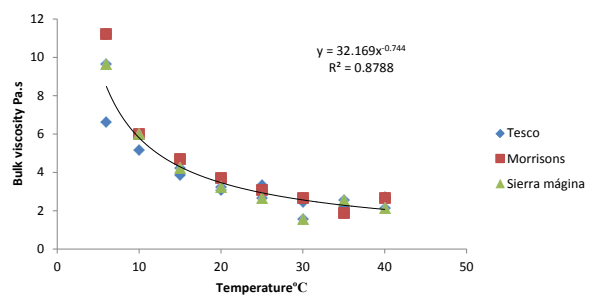

Figure 7: A plot of bulk viscosity v/s temperature of all the three brands (Tesco, Morrisons, Sierra magina) of extra-virgin olive oil. As there is no significant difference between the brands an averaged polynomial best fit is obtained from this plot to know the temperature dependence of bulk viscosity for extra-virgin olive oil.

there is a significant difference in the bulk viscosity values between the different brands of edible oils. Lastly, the temperature dependence of bulk viscosity was investigated (Figures 6 and 7).

\section{Materials and Methods}

Three brands of sunflower oil and three brands of extra-virgin olive oil have been used to evaluate potential variance of bulk viscosity with different brands of the same oil. The samples used for sunflower oil 
were Morrisons sunflower oil, Tesco sunflower oil and Floras sunflower oil. The samples used for olive oil was Morrisons extra virgin olive oil, Tesco extra virgin olive oil and Sierra mágina extra virgin olive oil. All the samples were locally purchased from Leeds supermarkets during July, 2015. Ultrasonic waves have been employed in this work to find the bulk viscosity. The attenuation coefficient, velocity of the sound wave after passing through the material across a range of frequencies is obtained from Ultrasizer MSV by Malvern Ltd. Density of the samples was measured by Anton Paar DMA 4500 M Density-meter. Anton Paar MCR 302 (Modular Compact Rheometer) was used to find the shear viscosity of the samples. All these parameters were measured to calculate the bulk viscosity using equation (8).

Ultrasizer MSV by Malvern Ltd was used to determine attenuation coefficient and velocity of sound as it passes through the oil samples at different selected temperatures. This is an acoustic spectroscopy instrument for liquids and emulsions operating in the frequency range of 1-100 MHz. Transducers are devices that convert energy from one form to other. This device makes use of two such transducers where one emits ultrasound waves on the application of voltage into the sample while the other detects it and converts into the corresponding voltage. Two pairs of such transducers are used. One pair operates in the low frequency range and the other in the high frequency range. On each run 50 measurements are taken covering the frequency range of 1 to $100 \mathrm{MHz}$. As sound waves travels through a medium attenuation is caused due to dissipation of energy in the form of shear viscosity, bulk viscosity, thermal conductivity and molecular relaxations. Ultrasizer measures this attenuation. This instrument needs $500 \mathrm{ml}$ of sample to measure which is a measure drawback for limited sample volumes. It is connected to an external Huber Ministat temperature control unit which operates in the range of $5^{\circ} \mathrm{C}$ to $50^{\circ} \mathrm{C}$. While conducting the experiment a stirrer constantly agitated the sample in order to reduce the thermal variation in the bulk sample, the speed of the stirrer can be adjusted. Care was taken so that no air bubbles were formed as these bubbles causes excess attenuation [11]. 10 repeat measurements were taken at each selected frequency and the mean was calculated to take into consideration any uncertainties or variations due to measurement.

The density measurements for the samples were accomplished by using Anton Paar DMA 4500 M Density-meter across a temperature range of $5^{\circ} \mathrm{C}$ to $40^{\circ} \mathrm{C}$. The measurements by this instrument is based on the oscillating U-tube method. The thermal control is provided by two integrated Pt100 platinum thermometers together with Peltier elements. Viscosity related errors are automatically corrected over the full range of sample viscosities by measuring the damping effect of the viscous sample followed by a mathematical correction of the density value. Error while measuring the shear viscosity may arise due to 'sample under filling, uncertainties in the gap size, viscous heating effects, wall-slip errors, edge failure and radial migration.'

Anton Paar MCR 302 was used to take the shear viscosity readings. This rheometer is driven by air bearing supported EC (Electrically Commutated) motor technology. This ensures accuracy over a wide viscosity range. It is a digital instrument using digital signal processing technology. It makes use of patented normal force sensor. It also makes use of some patented features for convenience and to increase the efficiency. The measuring system used for our measurements was CP50-2 (Conical plate with diameter $50 \mathrm{~mm}$ and angle $2^{\circ}$ ). The temperature is controlled by a water bath. The shear viscosity values from $25^{\circ} \mathrm{C}$ to $40^{\circ} \mathrm{C}$ were measured and values outside the measurement range were extrapolated.

Thermal properties of the materials to be utilised in calculating the bulk viscosity were obtained from literature [6]. The ratio of specific heats, $\gamma=C_{p} / C_{v}$ was found to be almost equal to unity. Hence, the term due to the thermal property term in equation (9) was neglected. The bulk viscosity value was calculated from equation (10) using the values of mean attenuation, density, shear viscosity and the velocity of ultrasound as measured in experimental procedures.

\section{Statistical analysis}

The mean, standard deviation and coefficient of variance of the bulk viscosity were calculated at each temperature of the selected temperature range. T-test of two samples assuming equal variances between each brand and also single factor Annova was performed for both sunflower and olive oil to evaluate the significant difference in the value of bulk viscosity between different brands of sunflower oil and olive oil. Microsoft Excel (XLS) was used for the statistical analysis performed.

\section{Results}

Tesco sunflower oil has been taken as an example to show all the calculations and graphs. The experimental calculations for the other samples have been provided in the appendix for clarity and brevity.

A graph of log attenuation v/s log frequency has been plotted as shown in Figure 2 and the best fit polynomial was obtained in the frequency of the form $f^{6}$, (where $\delta$ is the exponent). $\delta$ is found to be almost equal to $2(\delta=1.8344)$. A linear relationship between log attenuation and $\log$ frequency was seen (as regression coefficient $>$ 0.999) for all the samples at each temperature.

The bulk parameters (density, shear viscosity, velocity) measured and the bulk viscosity calculated for Tesco sunflower oil across a temperature range of $6^{\circ} \mathrm{C}$ to $40^{\circ} \mathrm{C}$ is summarised in Table 1 . The mean bulk viscosity and standard deviation has been calculated across the range of $12 \mathrm{MHz}-100 \mathrm{MHz}$. A graph of bulk viscosity against frequency was plotted to illustrate the dependence of bulk viscosity on the frequency (Figure 3). Frequency below $12 \mathrm{MHz}$ has not been included, as at this frequency range the attenuation value is too small due to molecular relaxations. The attenuation values obtained for 10 repeats of the same sample were averaged at each frequency. Repeated measurements of the same sample have been taken to increase the confidence level of calculating an accurate averaged value as exact value is not attainable at each time. These attenuation values were put into equation (10) along with the other parameters to calculate the bulk viscosity at each frequency. The standard deviation (SD) and coefficient of variation (CV) which is the ratio of the standard deviation by the mean were calculated. To find out about the nature of the fluid (Newtonian or non-Newtonian), a shear stress against strain diagram was obtained for Tesco sunflower oil (Figure 4). This shows an excellent linear relationship $\left(\mathrm{R}^{2}=1\right)$ between stress and strain for all the samples at the selected temperatures.

The ratio of bulk viscosity to shear viscosity was calculated in order to compare their values (Table 2). A graph of bulk viscosity and shear viscosity has been plotted to find their dependence on temperature (Figure 5). Statistical analysis performed on the different brands to find the existence of significant difference in the bulk viscosity value has been summarised in Table 3. The p-value obtained from single factor Anova between the different brands were $0.81(\mathrm{p} \geq 0.05)$ for olive oil, $0.17(p \geq 0.05)$ for sunflower oil. This suggests there is no statistical significant difference in the bulk viscosity value between the different brands of the same oil. This is further supported by the p-values ( $\geq$ 0.05 ) obtained from performing t-test of two samples assuming equal 
Citation: Ghosh S, Holmes M, Povey M (2017) Temperature Dependence of Bulk Viscosity in Edible Oils using Acoustic Spectroscopy. J Food Process Technol 8: 676. doi: 10.4172/2157-7110.1000676

Page 5 of 6

\begin{tabular}{|c|c|c|c|c|}
\hline \multirow{2}{*}{ Sunflower oil } & Samples & T-M & T-F & M-F \\
\cline { 2 - 5 } & $p$-value & 0.33 & 0.05 & 0.40 \\
\hline \multirow{2}{*}{ Olive oil } & Samples & T-M & T-S & M-S \\
\cline { 2 - 5 } & $p$-value & 0.51 & 0.77 & 0.74 \\
\hline
\end{tabular}

T: Tesco; M: Morrisons; F: Flora; S: Sierra mágina

Table 3: $p$-values from t-test of two samples assuming equal variances between each brand of sunflower oil and extra virgin olive oil.

\begin{tabular}{|c|c|c|c|c|c|c|}
\hline \multirow{2}{*}{$\begin{array}{c}\text { Temperature } \\
\left({ }^{\circ} \mathbf{C}\right)\end{array}$} & \multicolumn{2}{|c|}{ Tesco } & \multicolumn{2}{c|}{ Morrisons } & \multicolumn{2}{c|}{ Flora } \\
\cline { 2 - 7 } & $\begin{array}{c}\text { Mean } \times \\
\mathbf{1 0}^{-2} \mathbf{P a . s}\end{array}$ & $\begin{array}{c}\mathbf{S D} \times \mathbf{1 0}^{-2} \\
\mathbf{P a . s}\end{array}$ & $\begin{array}{c}\text { Mean } \times \\
\mathbf{1 0}^{-2} \mathbf{P a . s}\end{array}$ & $\begin{array}{c}\mathbf{S D} \times \mathbf{1 0}^{-\mathbf{2}} \\
\mathbf{P a . s}\end{array}$ & $\begin{array}{c}\text { Mean } \times \\
\mathbf{1 0}^{-2} \mathbf{P a} . \mathbf{s}\end{array}$ & $\begin{array}{c}\text { SD } \times \mathbf{1 0}^{-2} \\
\mathbf{P a . s}\end{array}$ \\
\hline 6 & 5.79 & 1.89 & 4.83 & 1.87 & 5.84 & 1.83 \\
\hline 10 & 4.51 & 1.59 & 3.8 & 1.65 & 4.67 & 1.61 \\
\hline 15 & 3.57 & 1.43 & 2.89 & 1.44 & 3.97 & 1.42 \\
\hline 20 & 2.91 & 1.21 & 2.3 & 1.18 & 3.39 & 1.19 \\
\hline 25 & 3.33 & 0.99 & 1.98 & 0.98 & 3.22 & 0.98 \\
\hline 30 & 2.77 & 0.86 & 1.97 & 0.87 & 3.62 & 0.83 \\
\hline 35 & 2.07 & 0.71 & 2.87 & 0.78 & 3.12 & 0.72 \\
\hline 40 & 2.36 & 0.6 & 2.07 & 0.6 & 3.26 & 0.61 \\
\hline
\end{tabular}

*Standard deviation SD

*Mean was taken for the bulk viscosity in the frequency range of $12 \mathrm{MHz}-100 \mathrm{MHz}$. the lowest temperature is decided by the operating limit of the instrument

Table 4: The bulk viscosity values of three different brands of sunflower oil at the selected temperature range.

\begin{tabular}{|c|c|c|c|c|c|c|}
\hline \multirow{2}{*}{$\begin{array}{c}\text { Temperature } \\
\left({ }^{\circ} \mathbf{C}\right)\end{array}$} & \multicolumn{2}{|c|}{ Tesco } & \multicolumn{2}{c|}{ Morrisons } & \multicolumn{2}{c|}{ Flora } \\
\cline { 2 - 7 } & $\begin{array}{c}\text { Mean } \times \mathbf{1 0} \\
2 \text { Pa.s }\end{array}$ & $\begin{array}{c}\text { SD } \times \mathbf{1 0}^{-2} \\
\text { Pa.s }\end{array}$ & $\begin{array}{c}\text { Mean } \times \\
\mathbf{1 0}^{-2} \text { Pa.s }\end{array}$ & $\begin{array}{c}\text { SD } \times \mathbf{1 0}^{-2} \\
\text { Pa.s }\end{array}$ & $\begin{array}{c}\text { Mean } \times \\
\mathbf{1 0}^{-2} \text { Pa.s }\end{array}$ & $\begin{array}{c}\text { SD } \times \mathbf{1 0}^{-2} \\
\text { Pa.s }\end{array}$ \\
\hline 8 & 6.62 & 2.25 & 11.22 & 3.8 & 9.64 & 3.35 \\
\hline 10 & 5.17 & 1.9 & 5.98 & 1.82 & 6.01 & 2.07 \\
\hline 15 & 3.87 & 1.54 & 4.72 & 1.48 & 4.22 & 1.63 \\
\hline 20 & 3.09 & 1.35 & 3.69 & 1.34 & 3.24 & 1.44 \\
\hline 25 & 3.34 & 1.23 & 3.07 & 1.19 & 2.68 & 1.22 \\
\hline 30 & 2.44 & 1.01 & 2.67 & 1.02 & 1.56 & 0.88 \\
\hline 35 & 2.2 & 0.85 & 1.88 & 0.84 & 2.58 & 0.85 \\
\hline 40 & 2.74 & 0.73 & 2.66 & 0.71 & 2.16 & 0.74 \\
\hline
\end{tabular}

Standard deviation SD

*Mean was taken for the bulk viscosity in the frequency range of $12 \mathrm{MHz}$ $100 \mathrm{MHz}$. The lowest temperature is decided by the operating limit of the instrument

Table 5: The bulk viscosity values of three different brands of olive oil at the selected temperature range.

\begin{tabular}{|c|c|c|c|}
\hline $\begin{array}{c}\text { Temperature } \\
\mathbf{T}\left({ }^{\circ} \mathbf{C}\right)\end{array}$ & $\begin{array}{c}\text { Density } \\
\left.\boldsymbol{\rho} \mathbf{( k g ~ m}^{-3}\right)\end{array}$ & Velocity $\mathbf{v}\left(\mathbf{m ~ s}^{-1}\right)$ & $\begin{array}{c}\text { Shear viscosity } \\
(\mathbf{n} \times \mathbf{1 0}-\mathbf{P a} \mathbf{s})\end{array}$ \\
\hline 6 & 928.96 & 1516.2 & 5.82 \\
\hline 10 & 926.29 & 1501.93 & 5.29 \\
\hline 15 & 922.84 & 1485.02 & 4.64 \\
\hline 20 & 919.41 & 1469.02 & 3.99 \\
\hline 25 & 915.82 & 1451.75 & 3.35 \\
\hline 30 & 912.51 & 1434.15 & 2.7 \\
\hline 35 & 909.16 & 1451.75 & 1.77 \\
\hline 40 & 905.82 & 1403.29 & 1.61 \\
\hline
\end{tabular}

Table 6: Measured parameters: density, velocity, shear viscosity of Morrisons sunflower oil at the selected temperatures.

\begin{tabular}{|c|c|c|c|}
\hline $\begin{array}{c}\text { Temperature } \\
\mathbf{T}\left({ }^{\circ} \mathbf{C}\right)\end{array}$ & $\begin{array}{c}\text { Density } \\
\boldsymbol{\rho} \mathbf{( k g ~ m}^{-3} \mathbf{)}\end{array}$ & $\begin{array}{c}\text { Velocity } \\
\left(\mathbf{m ~ s}^{-1} \mathbf{)}\right.\end{array}$ & $\begin{array}{c}\text { Shear viscosity } \\
(\mathbf{n} \times \mathbf{1 0}-\mathbf{P a} \mathbf{~} \mathbf{)})\end{array}$ \\
\hline 6 & 927.92 & 1513.26 & 4.93 \\
\hline 10 & 925.9 & 1502.03 & 4.53 \\
\hline 15 & 922.45 & 1485.02 & 3.87 \\
\hline 20 & 919.03 & 1467.22 & 3.21 \\
\hline 25 & 915.59 & 1451.6 & 2.48 \\
\hline 30 & 912.22 & 1435.59 & 1.49 \\
\hline
\end{tabular}

\begin{tabular}{|l|l|l|l|}
\hline 35 & 908.84 & 1419.59 & 1.33 \\
\hline 40 & 905.48 & 1403.52 & 0.76 \\
\hline
\end{tabular}

Table 7: Measured parameters: density, velocity, shear viscosity of Flora's sunflower oil at the selected temperatures.

\begin{tabular}{|c|c|c|c|}
\hline $\begin{array}{c}\text { Temperature } \\
\mathrm{T}\left({ }^{\circ} \mathrm{C}\right)\end{array}$ & $\begin{array}{c}\text { Density } \\
\rho\left(\mathbf{k g ~ m}^{-3}\right)\end{array}$ & $\begin{array}{l}\text { Velocity } v \\
\left(\mathrm{~m} \mathrm{~s}^{-1}\right)\end{array}$ & $\begin{array}{c}\text { Shear viscosity } \\
\left(\eta \times 10^{-2} \mathrm{~Pa} . \mathrm{s}\right)\end{array}$ \\
\hline 8 & 922.41 & 1505.02 & 6.26 \\
\hline 10 & 920.48 & 1495.16 & 5.83 \\
\hline 15 & 917.11 & 1477.55 & 5.12 \\
\hline 20 & 913.2 & 1462.53 & 4.41 \\
\hline 25 & 910.33 & 1445.98 & 3.26 \\
\hline 30 & 906.95 & 1429.56 & 3 \\
\hline 35 & 903.58 & 1413.53 & 2.53 \\
\hline 40 & 900.21 & 1397.39 & 1.56 \\
\hline
\end{tabular}

Table 8: Measured parameters: density, velocity, shear viscosity of Tesco extravirgin olive oil at the selected temperatures.

\begin{tabular}{|c|c|c|c|}
\hline $\begin{array}{c}\text { Temperature } \\
\mathbf{T}\left({ }^{\circ} \mathbf{C}\right)\end{array}$ & $\begin{array}{c}\text { Density } \\
\boldsymbol{\rho} \mathbf{( k g ~ m}^{-3} \mathbf{)}\end{array}$ & $\begin{array}{c}\text { Velocity } \\
\left(\mathbf{m ~ s}^{-1} \mathbf{)}\right.\end{array}$ & $\begin{array}{c}\text { Shear viscosity } \\
\left(\mathbf{n} \times \mathbf{1 0}^{-\mathbf{2}} \mathbf{P a . s}\right)\end{array}$ \\
\hline 6 & 920.88 & 1502.07 & 5.26 \\
\hline 10 & 919.52 & 1492.69 & 5.04 \\
\hline 15 & 916.07 & 1479.57 & 4.5 \\
\hline 20 & 912.65 & 1462.53 & 3.95 \\
\hline 25 & 909.23 & 1444.25 & 3.35 \\
\hline 30 & 905.84 & 1429.1 & 2.86 \\
\hline 35 & 902.44 & 1412.98 & 2.76 \\
\hline 40 & 899.06 & 1397.4 & 1.61 \\
\hline
\end{tabular}

Table 9: Measured parameters: density, velocity, shear viscosity of Morrisons extra-virgin olive oil at the selected temperatures.

variances between each brand (Table 3). As there is no significant difference in bulk viscosity between the brands, a generalised temperature dependence model of bulk viscosity was established by taking the best fit from the average plot of the three brands. The bulk viscosity values calculated for all the samples have been listed in Tables 4 and 5.

\section{Discussion}

\section{Justification of the use of frequency squared equation}

Since the exponent term $\delta$ was almost equal to $2(\delta=1.8344)$ (Figure 2 ), hence the use of frequency squared equation (equation 10) to find the bulk viscosity was justified. Attenuation is the result of both classical mechanisms (shear and bulk viscosity, thermal contributions) as well as due to molecular relaxations. The reason for $\delta$ being less than 2 is due to the occurrence of molecular relaxations which has not been accounted for in the equation (Tables 6-9). One of the most important reasons for molecular relaxations maybe due to the molecular rearrangements that occur during the compression of oil in the ultrasonic field [12]. There might be some error as excess attenuation due to the formation of air bubbles in the sample inside the Ultrasizer. Also, any thermal fluctuations during the measurement might result in some error as attenuation, density, velocity are dependent on temperature as explained earlier.

\section{Dependence of bulk viscosity on frequency}

The higher the value of CV the more dispersed is the data. CV for the mean bulk viscosity across the frequency range was found to be $\geq 0.05$ at each temperature (Table 1). This indicates that across the frequency range there is a variation in the bulk viscosity value which cannot be ignored. This suggests that the bulk viscosity values calculated for all 


\begin{tabular}{|c|c|c|c|}
\hline $\begin{array}{c}\text { Temperature } \\
\mathbf{T}\left({ }^{\circ} \mathbf{C}\right)\end{array}$ & $\begin{array}{c}\text { Density } \\
\boldsymbol{\rho} \mathbf{( k g ~ m}^{-3} \mathbf{)}\end{array}$ & $\begin{array}{c}\text { Velocity } \\
\left(\mathbf{m ~ s}^{-1} \mathbf{)}\right.\end{array}$ & $\begin{array}{c}\text { Shear viscosity } \\
(\mathbf{n} \times \mathbf{1 0}\end{array}$ \\
\hline 6 & 922.41 & 1506.8 & 6.05 \\
\hline 10 & 920.48 & 1494.07 & 5.67 \\
\hline 15 & 917.11 & 1479.5 & 5.03 \\
\hline 20 & 913.2 & 1461.05 & 4.4 \\
\hline 25 & 910.33 & 1445.72 & 3.77 \\
\hline 30 & 906.95 & 1429.1 & 3.2 \\
\hline 35 & 903.58 & 1412.98 & 2.27 \\
\hline 40 & 900.21 & 1397.4 & 2.05 \\
\hline
\end{tabular}

Table 10: Measured parameters: density, velocity, shear viscosity of Sienna mágina extra-virgin olive oil at the selected temperatures.

the selected temperatures are frequency dependent and not constant. Also, from Figure 3 it is seen that the bulk viscosity decreases with the increase in frequency for all temperatures, showing bulk viscosity is frequency dependent. This indicates the non-Newtonian behaviour for sunflower oil and olive oil over the selected temperature and frequency range, as for a fluid to be Newtonian the bulk viscosity must be constant for a selected frequency range [6]. However, from the stress and strain diagram obtained from Figure 4, edible oils are showing Newtonian nature as the shear stress is proportional to the shear strain.

\section{Comparison of bulk and shear viscosity}

The ratio of bulk viscosity to shear viscosity was found to be around 1 at all the selected temperatures (Table 2). This shows for edible oils the bulk viscosity is almost equal to its shear viscosity. However, the contribution of bulk viscosity to sound propagation due to nonNewtonian fluid seems to be quite less than due to Newtonian fluid as the bulk viscosity of water (a Newtonian fluid) was reported to be almost three times larger than its shear viscosity [13]. A plot of bulk and shear viscosity against temperature (Figure 5) shows there is a decrease in both the values with the increase in temperature indicating both are temperature dependent.

\section{Test of hypothesis}

The hypothesis tested that there is a significant difference between the different brands of the same edible oil is rejected based on the results obtained from the statistical analysis $(p \geq 0.05)$ (Table 3$)$. This shows bulk viscosity of edible oils do not seem to be much affected by small compositional differences. This is an important finding for future bulk viscosity studies as only one brand can be used to represent a class of oil, saving both time and resources (Table 10).

\section{Temperature dependence of bulk viscosity}

A decrease in the bulk viscosity is seen as the temperature increases (Figures 6 and 7).

The temperature dependent bulk viscosity model has been established as:

$$
\mu=\mu_{1}[\mathrm{~T} \exp (\mathrm{A})]
$$

Where $\mu$ (Pa.s) is the bulk viscosity, $\mu_{1}$ (Pa.s. $\left.{ }^{\circ} \mathrm{C}^{-1}\right) \mathrm{T}$ is temperature $\left({ }^{\circ} \mathrm{C}\right), \mathrm{A}$ is constant, $\mu_{1}$ is 0.1092 and $\mathrm{A}$ is -0.413 for sunflower oil (Figure
6) and $\mu_{1}$ is 32.169 and $\mathrm{A}$ is -0.744 for extra-virgin olive oil (Figure 7). The findings from this study cannot be generalised for edible oils as the sample size was not adequate as only two types of oil: sunflower oil and extra-virgin olive oil was investigated and only three brands from each were taken into consideration. Further studies should be conducted with larger sample size like with other commonly used edible oils. For the different brands simple random sampling must be undertaken so that the samples well represents the entire class.

\section{Conclusion}

The temperature dependence of bulk viscosity in edible oil using acoustic spectroscopy was established using a model equation. The mean bulk viscosity decreases with the increase in temperature. The value of bulk viscosity is not constant over the frequency range, it decreases with increase in frequency. Therefore, in terms of bulk viscosity edible oils are non-Newtonian fluids. There is no significant statistical difference of bulk viscosity value between different brands of the same oil. This suggests that future studies with only one variety of oil will be enough. Even though other physical properties of edible oils have been extensively studied, little research has been done on bulk viscosity. More research should be undertaken to check the reproducibility of these results and validate the data.

\section{Acknowledgement}

I would like to thank the entire School of Food Science, University of Leeds and my supervisor Dr. Melvin Holmes for his constant support and guidance.

\section{References}

1. McClements DJ, Povey MJW (1992) Ultrasonic analysis of edible fats and oils Ultrasonics 30: 383-388.

2. Leighton TG (2007) What is ultrasound? Prog Biophys Mol Biol 93: 3-83.

3. Morrison FA (2004) Compressible fluids. Michigan Technological University, USA pp: 94-98.

4. Hirai N, Eyring H (1958) Bulk viscosity of liquids. J Appl Phys 29: 810

5. Baidakov VG, Protsenko SP (2014) Metastable Lennard-Jones fluids: III. Bulk viscosity. J Chem Phys 141: 114503.

6. Dukhin AS, Goetz PJ (2009) Bulk viscosity and compressibility measurement using acoustic spectroscopy. J Chem Phys 130: 124519.

7. Cramer MS, Bahmani F (2014) Effect of large bulk viscosity on large-Reynolds number flows. J Fluid Mechanic 751: 142-163.

8. Gladwell N, Javanaud C, Peers KE, Rahalkar RR (1985) Ultrasonic behavio of edible oils: Correlation with rheology. J Am Oil Chem Soc 62: 1231-1236.

9. Coupland JN, McClements DJ (1997) Physical properties of liquid edible oils. $J$ Am Oil Chem Soc 74: 1559-1564

10. Rodrigues Jr. JDA, Cardoso FDP, Lachter ER, Estevao LRM, Lima E, et al. (2006) Correlating chemical structure and physical properties of vegetable oil esters. J Am Oil Chem Soc 83: 353-357.

11. Dalen J, Lo A (1981) The influence of wind-induced bubbles on echo integration surveys. J Acou Soc Am 69: 1653-1659.

12. Chanamai R, McClements DJ (1998) Ultrasonic attenuation of edible oils. J Am Oil Chem Soc 75: 1447-1448.

13. Holmes M, Parker NG, Povey MJW (2011) Temperature dependence of bulk viscosity in water using acoustic spectroscopy. J Phys Conf Ser. 